\title{
DARK MATTER POLARIZATION OPERATOR IN THE GENERALIZED YUKAWA MODEL
}

\author{
M.S. Dmytriiev, V.V. Skalozub \\ Oles’ Honchar Dnipro National University, Dnipro, Ukraine \\ e-mail: dmytrijev_m@ffeks.dnu.edu.ua
}

\begin{abstract}
Nowadays, no dark matter candidates have been discovered. We consider the possible reason for that which is related to the approach of on-peak resonance searching for. As is believed usually, a new particle has small width and a narrow width approximation is applicable to identify such type resonant peak in the invariant mass spectrum of collision products.

In the present paper, in the framework of the generalized Yukawa model, we find out the properties of the searched particle when its width is larger than a maximal one expected during experiments and so this state could be missed as a noise. Usually, the new particle width is considered as an arbitrary parameter. Here, we obtain the width of the dark matter particle from an imaginary part of polarization operators. Then the width is analyzed as explicit function of the couplings and masses in the underlying model of the dark matter. The corresponding constraints on the model parameters are obtained. Role of the one-loop mixing of visible and dark matter fields is investigated and constraint on the mixing angle value is derived. These estimations are quite general and, in particular, relevant to interactions between the particles of the Standard model and dark matter.
\end{abstract}

Keywords: dark matter, resonance, narrow width approximation, mixing of fields.

Received 12.07.2020; Received in revised form 15.08.2020; Accepted 01.09.2020

\section{Introduction}

Nowadays, no particles-candidates for dark matter (DM) have been found, yet. There is a long list of such particles entering different models relevant to various energy scales. In what follows, we consider why it could be so within the standard treating of scattering experiment data. It is usually assumed that resonances of the unknown particles are narrow ones and their typical width $\Gamma$ is about $1-3 \%$ of the peak's mass $\mathrm{M}$, so $\Gamma \ll \mathrm{M}$. This assumption allows us to apply the NWA to discover the resonances in the total cross-section. In this approach, the interference between visible and dark particles could be neglected in the total cross section. At the same time, in the literature there are many models beyond the Standard model (SM), which anticipate new particles to have big widths of resonances. However, such type states can be missed as a noise if they appear in experimental data. In this paper we analyze how the widths of new physics peaks depend on masses and couplings introduced in some underlying model of the DM. This research is also motivated by [1], where constraints on the model parameters of the hypothetical Z' boson are considered. It is found there that a small mixing angle between the SM Z boson and Z' allows the latter to have the width of up to $100 \%$ of its mass. Similarly, if the dark matter particle has small mixing with the visible matter, its resonance could also be wide, without any effect on the properties of visible matter. We are interested in the model when the dark particle acquires a large width.

For that we introduce the generalized Yukawa model. DM is presented as the heavy Dirac fermion $\Psi$ and scalar $\chi$ fields. Visible matter is presented as the light scalar field $\varphi$ and the doublet of light fermions $\psi_{1}$ and $\psi_{2}$. The latter two interact with bosons $\varphi$ and $\chi$ through different Yukawa's couplings. Using this model, we obtain the width of dark boson $\chi$ for certain values of the model parameters. Such approach is different from that used in some non-NWA peak investigations presented in, for instance, $[2,3,4]$. In these papers the width of the new particle is adopted to be an arbitrary free parameter. In our research, we obtain the explicit analytic expression for the width of the dark particle, as a function of model couplings and masses. 
There exist numerous models of DM such as SUSY particles (neutralinos) [5], neutral Z' bosons [6, 7, 8], sterile neutrinos [9], etc. In these models, it is assumed that the DM candidate has a certain group of symmetry or specified couplings to other fields. On the contrary, we consider all the parameters of DM as free, and do not limit our treatment by a certain gauge group.

Below we are concentrating on the analysis of the particle parameter values for which the dark resonance becomes invisible in the direct search for it. Then we derive necessary constraints on the properties of the DM sector.

The paper is organized as follows. In next section we introduce our model and discuss the radiation corrections to the boson masses, alongside with the mixing of scalar fields, which appears at one-loop level. In sect. 3 we define the corresponding mixing angle. In sect. 4, we consider the width of the dark particle resonance and its analytical properties as the function of model parameters. Then we estimate the values of the parameters when this width exceeds the search limits, and provide the limitations for the mixing angle. We summarize our results in the context of comparisons with a number of DM models in the last section.

\section{Radiation corrections to the boson masses}

We start with the Lagrangian

$$
\begin{aligned}
& \mathscr{L}=\frac{1}{2}\left[\left(\partial_{\mu} \phi\right)^{2}-\mu^{2} \phi^{2}\right]+\frac{1}{2}\left[\left(\partial_{\mu} \chi\right)^{2}-\Lambda^{2} \chi^{2}\right]-\lambda \phi^{4}-\rho \phi^{2} \chi^{2}-\xi \chi^{4}+ \\
& +\sum_{a=1 ; 2} \bar{\psi}_{a}\left(i \gamma^{\mu} \partial_{\mu}-g_{\phi} \phi-g_{\chi} \chi-m_{a}\right) \psi_{a}+\bar{\Psi}\left(i \gamma^{\mu} \partial_{\mu}-M-G_{\chi} \chi\right) \Psi .
\end{aligned}
$$

Dark fermions $\Psi$, having the coupling $\mathrm{G}_{\chi}$ to scalars in the dark sector, do not interact with visible bosons $\varphi$. The visible fermions are coupled to both visible and dark scalars.

The fields $\varphi$ and $\chi$ acquire their widths through the radiation corrections giving imaginary parts to their masses. Such corrections appear due to the interactions of scalars with themselves and the fermions. The loop corrections for the field $\varphi$ we denote as $\Pi_{\varphi \varphi}\left(\mathrm{p}^{2}\right)$, where $\mathrm{p}^{2}$ is the squared momentum transferred through the loop. Similarly, corrections for the field $\chi$ are denoted as $\Pi_{\chi \chi}\left(\mathrm{p}^{2}\right)$. Diagrams contributing to the $\Pi_{\varphi \varphi}\left(\mathrm{p}^{2}\right)$ and $\Pi_{x x}\left(\mathrm{p}^{2}\right)$ are shown in Figs. 1 and 2, respectively. There is also the diagram of scalar field mixing which contribution is denoted as $\Pi_{\varphi \chi}\left(\mathrm{p}^{2}\right)$ and shown in Fig. 3.
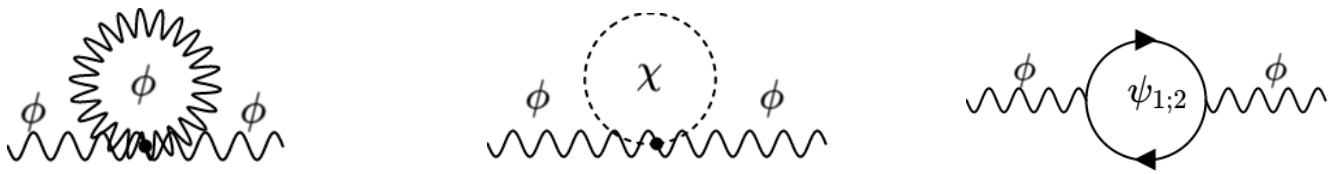

Fig. 1. Loop corrections contributing to $\Pi_{\varphi \varphi}\left(p^{2}\right)$.
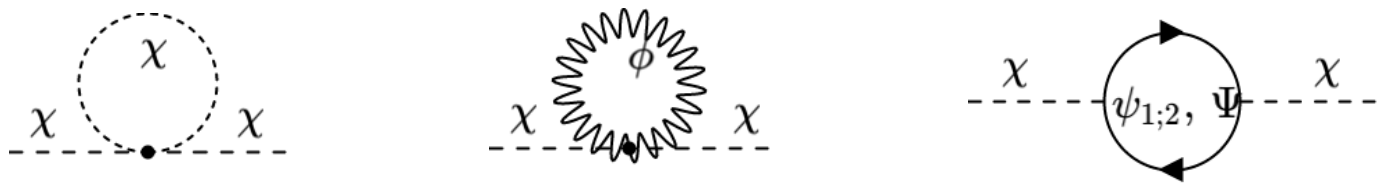

Fig. 2. Loop corrections contributing to $\Pi_{\chi \chi}\left(p^{2}\right)$. 


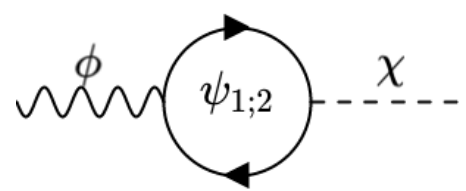

Fig. 3. The loop correction contributing to $\Pi_{\varphi x}\left(p^{2}\right)$.

The definitions of the two-point Green functions of the scalar fields in the Heisenberg representation read:

$$
\begin{aligned}
& G_{\phi \phi}\left(x_{1} ; x_{2}\right)=\left\langle 0\left|T \phi\left(x_{1}\right) \phi\left(x_{2}\right)\right| 0\right\rangle, \quad G_{\phi \chi}\left(x_{1} ; x_{2}\right)=\left\langle 0\left|T \phi\left(x_{1}\right) \chi\left(x_{2}\right)\right| 0\right\rangle, \\
& G_{\chi \chi}\left(x_{1} ; x_{2}\right)=\left\langle 0\left|T \chi\left(x_{1}\right) \chi\left(x_{2}\right)\right| 0\right\rangle .
\end{aligned}
$$

From the Schwinger-Dyson equation, Green functions in Eq. (2) are expressed through geometric series of one-particle irreducible diagrams. Due to the one-loop mixing effect, the Schwinger-Dyson equation is actually a matrix expression. In the Fourier representation, the Green functions read (momentum arguments are omitted for brevity):

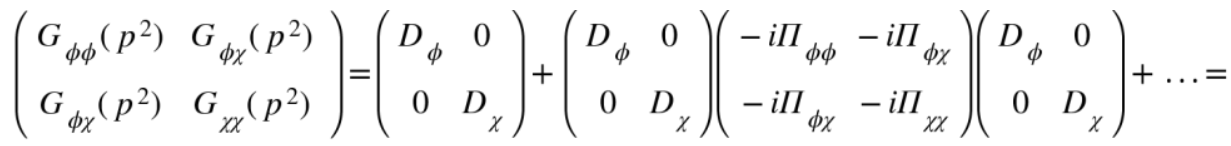

$$
\begin{aligned}
& =i\left(\begin{array}{cc}
p^{2}-\mu^{2}-\Pi_{\phi \phi}\left(p^{2}\right) & -\Pi_{\phi \chi}\left(p^{2}\right) \\
-\Pi_{\phi \chi}\left(p^{2}\right) & p^{2}-\Lambda^{2}-\Pi_{\chi \chi}\left(p^{2}\right)
\end{array}\right)^{-1}, \\
& D_{\phi}=D_{\phi}\left(p^{2}\right)=\frac{i}{p^{2}-\mu^{2}} \quad D_{\chi}=D_{\chi}\left(p^{2}\right)=\frac{i}{p^{2}-\Lambda^{2}} .
\end{aligned}
$$

In this equation, $\mathrm{D}_{\varphi}\left(\mathrm{p}^{2}\right)$ and $\mathrm{D}_{\chi}\left(\mathrm{p}^{2}\right)$ are the propagators of the free scalar fields. Functions $\Pi_{\varphi \varphi}\left(\mathrm{p}^{2}\right), \Pi_{\gamma \chi}\left(\mathrm{p}^{2}\right)$ and $\Pi_{\varphi \chi}\left(\mathrm{p}^{2}\right)$ are components of the vacuum polarization operator $\Pi\left(\mathrm{p}^{2}\right)$ :

$$
\Pi\left(p^{2}\right)=\left(\begin{array}{ll}
\Pi_{\phi \phi}\left(p^{2}\right) & \Pi_{\phi \chi}\left(p^{2}\right) \\
\Pi_{\phi \chi}\left(p^{2}\right) & \Pi_{\chi \chi}\left(p^{2}\right)
\end{array}\right) .
$$

We perform calculation in a renormalized perturbation theory, and the polarization operator components are renormalized by the counter terms in eq. (5). These counter terms cancel divergences in the components of $\Pi\left(\mathrm{p}^{2}\right)$ from the vacuum polarization diagrams in Figs. 1, 2, 3:

$$
\begin{aligned}
& \Pi_{\phi \phi}^{(f i n)}\left(p^{2}\right)=\Pi_{\phi \phi}\left(p^{2}\right)-\left(p^{2} \delta_{\phi \phi}-\delta_{\mu}\right) \\
& \Pi_{\chi \chi}^{(f i n)}\left(p^{2}\right)=\Pi_{\chi \chi}\left(p^{2}\right)-\left(p^{2} \delta_{\chi \chi}-\delta_{\Lambda}\right) \\
& \Pi_{\phi \chi}^{(f i n)}\left(p^{2}\right)=\Pi_{\phi \chi}\left(p^{2}\right)-\left(p^{2} \delta_{\phi \chi}-\delta_{\kappa}\right)
\end{aligned}
$$

In eq. (5) the superscript (fin) denotes a renormalized polarization operator. Hereafter we omit this superscript, considering only finite parts of the polarization operator components. To regularize loop integrals a dimensional regularization is used. The diverging parts of the integrals are subtracted according to the $\overline{M S}$ scheme. The counter terms in eq. (5) are chosen to fulfill the renormalization conditions:

$$
\mathfrak{R} \Pi_{\phi \phi}\left(p^{2}=\mu^{2}\right)=0, \quad \mathfrak{R} \Pi_{\chi \chi}\left(p^{2}=\Lambda^{2}\right)=0, \quad \mathfrak{R} \Pi_{\varphi \chi}\left(p^{2}=\kappa^{2}\right)=0 .
$$


Hence, $\mu^{2}$ and $\Lambda^{2}$ are the real parts of the poles of the Green functions matrix in the set (3). Parameter $\kappa^{2}$ is chosen arbitrarily, to renormalize the one-loop mixing diagram in Fig. 3. According to the conditions (6), scalar loop contributions into $\Pi_{\varphi \varphi}\left(\mathrm{p}^{2}\right)$ and $\Pi_{\chi \chi}\left(\mathrm{p}^{2}\right)$ are completely absorbed by the counter terms.

\section{Width of DM particles}

In the on-resonance searches, the new particle is identified with the resonant peak in the cross-section of some scattering process. The resonance position coincides with the mass.

Width of $\chi$ particle is defined by the imaginary part of its polarization operator $\Pi_{\chi x}\left(\mathrm{p}^{2}\right)$ taken at the point $\mathrm{p}^{2}=\Lambda^{2}$. Here $\mathrm{p}^{2}$ is the squared momentum transferred through the virtual boson state. $\Pi_{\chi x}\left(\mathrm{p}^{2}\right)$ is calculated analytically, and its imaginary part is

$$
\begin{aligned}
& \Im \Pi_{\chi \chi}\left(p^{2}\right)=\frac{p^{2}}{8 \pi}\left\{g_{\chi}^{2}\left[\left(1-\frac{4 m_{1}^{2}}{p^{2}}\right)^{\frac{3}{2}}+\left(1-\frac{4 m_{2}^{2}}{p^{2}}\right)^{\frac{3}{2}}\right]+G_{\chi}^{2}\left(1-\frac{4 M^{2}}{p^{2}}\right)^{\frac{3}{2}}\right\}, \\
& p^{2} \geq \max \left(4 m_{1}{ }^{2} ; 4 m_{2}^{2} ; 4 M^{2}\right) .
\end{aligned}
$$

Hence, the width $\rho$ of the $\chi$ resonance, as a fraction of mass $\Lambda$ equals:

$$
\begin{aligned}
& \rho=\frac{\Gamma}{\Lambda}=\frac{\Im \Pi_{\chi \chi}\left(\Lambda^{2}\right)}{\Lambda^{2}}= \\
& =\frac{g_{\chi}^{2}}{8 \pi}\left[\left(1-\frac{4 m_{1}^{2}}{\Lambda^{2}}\right)^{\frac{3}{2}}+\left(1-\frac{4 m_{2}^{2}}{\Lambda^{2}}\right)^{\frac{3}{2}}\right]+\frac{G_{\chi}^{2}}{8 \pi}\left(1-\frac{4 M^{2}}{\Lambda^{2}}\right)^{\frac{3}{2}} .
\end{aligned}
$$

Below we plot $\rho$ as the function of variables $g_{\chi}, G_{\chi}$ and $\Lambda$, in Figs. 4 and 5. The plots are contour maps of the $\chi$ widths with the specific contour highlighted by the solid dashed line, at which $\rho=3 \%$. This contour separates the areas in the parametric space, where $\rho$ $<3 \%$ (narrow peaks) and where $\rho>3 \%$ (wide peaks). Narrow resonances are potentially visible in the experiment. In all these figures the parameter $M$ is taken to be the same $\left(\mathrm{M} \approx 2 \cdot 10^{3} \mathrm{~m}_{1}\right)$.
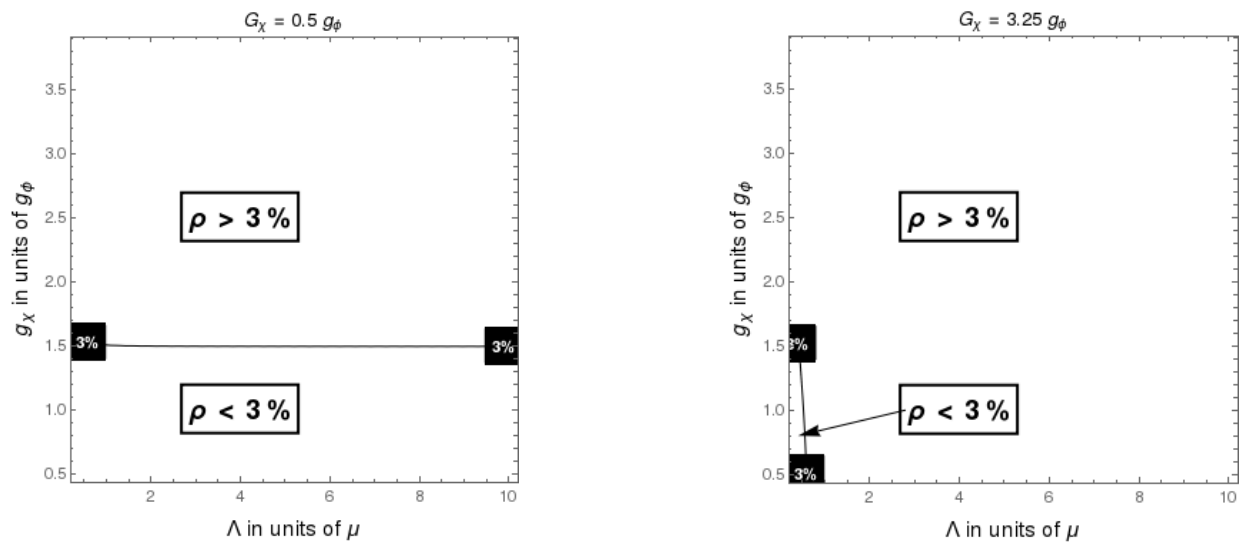

Fig. 4. Contour maps of dark boson widths in the parametric space $\Lambda$ - $g_{\chi}$, with fixed $G_{\chi}$. Labels describe regions with the appropriate widths values.

From graphs in Figs. 4 and 5 we identify, whether the resonance is wide or narrow, for the given values of the model parameters. 
As we can see from the second graph in Fig. 4, if the new boson interacts sufficiently strong with other particles inside the dark sector, the formation of new narrow resonance is rather exclusive than typical. Namely, this is so when the new particle is lighter than the known one. For example, in Fig. 4, in the second graph, for $\mathrm{g}_{\chi} \approx \mathrm{g}_{\varphi}$, we have that resonance is narrow until $\Lambda \lesssim 0,5 \mu$. Such boson is easily detectable. So that it can be rejected. For $G_{\chi} \gg g_{\varphi}$ we find that if new particle is heavier than the visible one, its peak has to be wide. Contrary to this, if $\chi$ and $\Psi$ interact weakly, the new peak is narrow one in a wide range of its mass and for coupling $\mathrm{g}_{\chi} \approx \mathrm{g}_{\varphi}$. In the latter case, if $\Lambda \leq \mu$, the dark peaks are narrow for almost whole range of $G_{\chi}$ variation (Fig. 5). But if $g_{\chi} \gg g_{\varphi}$ all dark resonances are wide states. As we can see from the last graph in Fig. 5, the narrow peaks do not exist in that case. Hence, the DM should have sufficiently strong interaction inside the dark sector or with the visible matter, to produce wide resonance. Finally, to keep the wide dark resonance, there has to be $\Lambda>\mu$ and either $g_{\chi} \gg g_{\varphi}$ or $G_{\chi} \gg g_{\varphi}$.

According to the results of the modern experiments, new hypothetical bosons beyond the Standard model do not change properties of known resonances [8]. It is so if the masses of two resonances are far enough one from another, and they do not intersect. Hence, the found condition $\Lambda>\mu$ is particularly important - if it is not satisfied, the dark particle would be detected.
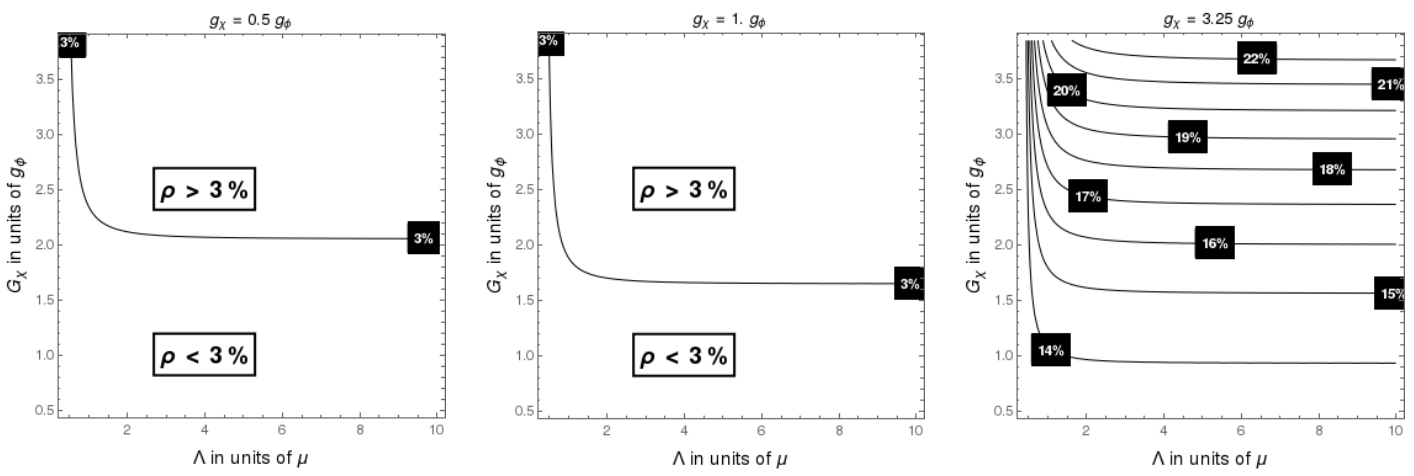

Fig. 5. Contour maps of dark boson widths in the parametric space $\Lambda$ - $G_{\chi}$, with fixed $g_{\chi}$. Labels describe regions with the appropriate widths values.

To summarize, we have investigated the influence of the mass $M$ of dark fermion on the $\chi$-particle width. It is worth noting that the existence of additional fermions beyond the visible sector is not obligatory. This is because only the properties of the dark boson particle are under consideration. Nevertheless, the condition of wide $\chi$ resonance sets certain restrictions on the $\Psi$-field mass. It was found that when mass $M$ is increased the DM peak is narrowing. Hence, to keep the width of $\chi$ bigger than $3 \%$, we anticipate dark fermions to have the mass values in the scale of visible ones. The estimate of the $\mathrm{M}$ upper bound is, $\mathrm{M} \leq 10^{3}-10^{4} \mathrm{~m}_{1}$. We also note that visible fermions were considered to be light enough to contribute the boson widths, so that $2 \mathrm{~m}_{1 ; 2} \ll \Lambda$.

\section{Discussion and conclusion}

Above we show how the DM particle acquires its width through the interaction with other fields of the model. Analytically its resonance width depends on the contributions from the interactions with the model fermions. These contributions are defined both by the corresponding Yukawa couplings and mass of the resonance. The self-interaction of bosons does not affect its width, being canceled in the renormalization procedure. These results are general and do not depend on the transformation properties of the dark boson field. 
In the previous section we have analyzed the role of values of the particle couplings and the masses resulting in the creation of wide resonances in scattering processes. Such type resonances could not be detected by the standard methods of direct searches of the resonances which have $\rho<3 \%$. To realize that, we divided the fields into "visible" and "dark" ones and considered various scenarios ensuring the DM boson gains a large resonance width in the invariant mass spectrum of final states. It turns out that the limit of $3 \%$ can be exceeded in many cases. In the framework of our model, the conditions for that are the following:

1. DM particle is heavier than the visible one $-\Lambda>\mu$

2. Interactions in the visible sector are weaker than that of between the dark and visible particle or between the particles in the dark sector, only. That is, if either $\mathrm{g}_{\chi} \gg \mathrm{g}_{\varphi}$ or $\mathrm{G}_{\chi} \gg \mathrm{g}_{\varphi}$.

It also can be shown that wide resonances correspond to the small mixing between dark and visible fields. We find that the mixing angle obtained from the bosonic mass matrix in the effective potential of scalars has to be less than $10^{-5}$. The presence of this upper limit is qualitatively important. If the mixing angle is small, dark boson resonance also does not affect the visible sector properties.

The considered Yukawa model gives a possibility for analyzing the role of the masses and couplings of particles. Other aspects of the problem such as group symmetry of the extended model and, hence, the content of the states remain behind it. However, we have obtained the set of conditions which have to be taken into account when searches for the DM particles are carried out. In general, to avoid the problem of wide resonance states we have to apply additionally non-resonant methods to detect these new states of matter. Among them, the interference of dark and visible states should be taken into consideration at energies far from resonance peak. Different types of the effective Lagrangians could be derived to describe interactions between two worlds. These are problems left for the future.

\section{References}

1. Pevzner, A. Estimate of the abelian Z' decay width / A. Pevzner // Visnik Dnipropetrovs'kogo universitetu. Seria Fizika, radioelektronika. - 2017. - Vol. 25. P. 7-13

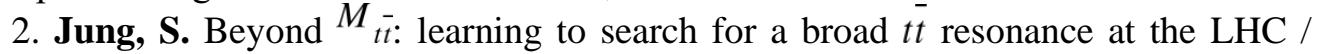
Sunghoon Jung, Dongsub Lee, Ke-Pan Xie // Eur. Phys. J. - 2020. - C 80:105

3. Moretti, S. Production of extra quarks at the Large Hadron Collider beyond the narrow width approximation / Stefano Moretti, Dermot O'Brien, Luca Panizzi, Hugo Prager // Phys. Rev. -2017.- D 96, 075035

4. Moretti, S. Production of extra quarks decaying to dark matter beyond the narrow width approximation at the LHC / Stefano Moretti, Dermot O'Brien, Luca Panizzi and Hugo Prager // Phys. Rev. -2017.- D 96, 035033

5. Drees, M. Neutralino Dark Matter in Scenarios with Early Matter Domination / Manuel Drees, Fazlollah Hajkarim // JHEP -2018.- 1812042

6. Leike, A. The Phenomenology of Extra Neutral Gauge Bosons / A. Leike. // Phys. Rept. -1999.- 317:143-250.

7. Langacker, P. The Physics of Heavy Z' Gauge Bosons / Paul Langacker // Rev. Mod. Phys. -2009.- 81:1199-1228

8. Gulov, A. Global search for the Z' boson at Modern Colliders / A. Gulov, V. Skalozub // (Lira Ltd., Dnipro, 2020), p. 140.

9. Boyarsky, A. Sterile Neutrino Dark Matter / A. Boyarsky, M. Drewes, T. Lasserre, S. Mertens, O. Ruchayskiy // e-print arXiv:1807.07938 [hep-ph]. 